\title{
RANK ELEMENT OF A PROJECTIVE MODULE
}

\author{
AKIRA HATTORI
}

In $\S 1$ of this note we first define the trace of an endomorphism of a projective module $P$ over a non-commutative ring $A$. Then we call the trace of the identity the rank element $r(P)$ of $P$, which we shall illustrate by several examples. For a projective module $P$ over the groupalgebra of a finite group $G$, the rank element of $P$ is essentially the character of $G$ in $P$. In $\S 2$ we prove that under certain assumption two projective modules $P_{1}$ and $P_{2}$ over an algebra over a complete local ring $D$ are isomorphic if and only if their rank elements are identical. This is a type of proposition asserting that two representations are equivalent if and only if their characters are identical, and in fact, when $A$ is the groupalgebra, the above theorem may be considered as another formulation of Swan's local theorem $\left.[9]^{*}\right)$.

\section{§1. Trace and rank element}

1.1. Let $A$ be a ring with an identity, and $[A, A]$ its commutator, i.e. the set of finite sums of elements of the form $a b-b a(a, b \in A)$. We denote the abelian group $A /[A, A]$ by $A^{a}$, and the natural epimorphism $A \rightarrow A^{a}$ by $\varepsilon$. $\varepsilon$ is symmetric: $\varepsilon(a b)=\varepsilon(b a)$.

For a left $A$-module $M$, we have the right $A$-module $\operatorname{Hom}_{A}(M, A)$, called the dual module of $M$ and denoted by $M^{*}$ in this paper. It is easy to check that the pairing $(\xi, x) \rightarrow \varepsilon(\xi(x))\left(\xi \in M^{*}, x \in M\right)$ induces a well-defined homomorphism $M^{*} \otimes_{\triangle} M \rightarrow A^{a}$, which we shall denote by $\pi$.

Let $P$ be a finitely generated projective left $A$-module. This means that the mapping $\theta: P^{*} \otimes{ }_{A} P \rightarrow \operatorname{Hom}_{A}(P, P)$ defined by $\theta(\xi \otimes x)(y)=\xi(y) x(x, y \in P$, $\xi \subseteq P^{*}$ ) is an isomorphism. Now, we shall define the trace $\operatorname{Tr}_{A}(f)$ (or simply $\operatorname{Tr}(f))$ of an endomorphism $f \in \operatorname{Hom}_{A}(P, P)$ by $\operatorname{Tr}(f)=\pi\left(\theta^{-1}(f)\right)$, which is a

Received January 25, 1964.

*) The present work was done in 1960-1961, and briefly announced in [5]. Our main objective was to study Swan's results without use of Grothendieck rings. In the meantime, there appeared the works of Giorgiutti [4], Rim, and Bass [2], which gave the same problem nice answers together with a generalization. 
well-defined element of $A^{a}$. If $\theta^{-1}(1)=\sum \xi_{i} \otimes x_{i}$, then $\theta^{-1}(f)=\sum \xi_{i} \otimes f\left(x_{i}\right)$. It follows that $\operatorname{Tr}(f)=\varepsilon \sum \xi_{i}\left(f\left(x_{i}\right)\right)$.

If $g$ is another endomorphism of $P$, we have

$$
\begin{aligned}
\operatorname{Tr}(f g) & =\varepsilon \sum \xi_{j}\left(g\left(x_{i}\right)\right) \xi_{i}\left(f\left(x_{j}\right)\right) \\
& =\varepsilon \sum \xi_{i}\left(f\left(x_{j}\right)\right) \xi_{j}\left(g\left(x_{i}\right)\right)=\operatorname{Tr}(g f) .
\end{aligned}
$$

Hence $\operatorname{Tr}$ is a symmetric additive mapping. If $A$ is commutative and $P$ is free, then our trace is identical with the usual trace of $f$ or of the corresponding matrix.

By a further analogy, we call $\operatorname{Tr}(1)$ the rank element of the projective module $P$, and denote it by $r_{A}(P)$ (or by $r(P)$ ). Clearly we have $r\left(P_{1} \oplus P_{2}\right)=$ $r\left(P_{1}\right)+r\left(P_{2}\right)$. If $P$ is free of rank $n$, then $r(P)=\varepsilon(n \cdot 1)$.

EXAMPLES 1. Let $o$ be an integral domain, and $a$ an invertible (= projective) ideal of 0 . There exist $a_{i} \in \mathfrak{a}$ and $b_{i} \in \mathfrak{a}^{-1}(i=1, \ldots, n)$ such that $\sum a_{i} b_{i}=1$. For $f \in \operatorname{Hom}_{\mathfrak{D}}(a, a)$, we have $\operatorname{Tr}(f)=\sum b_{i} f\left(a_{i}\right)$. It follows $\operatorname{Tr}(f) a=\sum\left(a b_{i}\right) f\left(a_{i}\right)$ $=f\left(a \sum b_{i} a_{i}\right)=f(a)$ for $a \in \mathfrak{a}$. In particular, $r_{\mathrm{D}}(\mathfrak{a})=\operatorname{Tr}(1)=1$.

2. Let $A=\mathfrak{I}_{1} \oplus \mathfrak{I}_{2}$ be a decomposition of $A$ into a direct sum of left ideals, and $1=e_{1}+e_{2}$ the corresponding decomposition of 1 into the sum of orthogonal idempotents. Put $P=l_{1}=A e_{1}$. Then $\theta^{-1}(1)=\ell_{1} \otimes e_{1}$, where $\iota_{1}$ is the natural injection $l_{1} \rightarrow A$. Hence we have $\operatorname{Tr}(f)=\varepsilon\left(f\left(e_{1}\right)\right)$. In particular, $r_{A}\left(l_{1}\right)=\varepsilon\left(e_{1}\right)$. Let $e$ and $e^{\prime}$ be idempotents, and assume that there is an $A$-isomorphism $\varphi: A e$ $\cong A e^{\prime}$. If $\varphi(e)=a^{\prime} e^{\prime}$ and $\varphi^{-1}\left(e^{\prime}\right)=a e$, then we have $e=a^{\prime} e^{\prime} a e$ and $e^{\prime}=a e a^{\prime} e^{\prime}$. Hence $\varepsilon(e)=\varepsilon\left(e^{\prime}\right)$.

3. Let $A$ be a separable algebra over $R$ (Auslander-Goldman [1]). This means that there exists $\sum u_{i} \otimes v_{i}^{\circ} \in A^{e}=A \otimes A^{\circ}$ (where $A^{\circ}$ is an anti-isomorphic copy of $A$ ) satisfying the following i) and ii) (cf. e.g. Hattori [6]) :

i) $\sum u_{i} v_{i}=1$,

ii) $\sum a u_{i} \otimes v_{i}^{\circ}=\sum u_{i} \otimes\left(v_{i} a\right)^{\circ} \quad$ for any $a \in A$.

Define a linear mapping $s: A \rightarrow A$ by $s(x)=\sum v_{i} x u_{i}$. By ii) we have

$$
s(x y)=\sum v_{i} x\left(y u_{i}\right)=\sum\left(v_{i} y\right) x u_{i}=s(y x) .
$$

Conversely, if $s(z)=0$, then by i) we have

$$
z=\sum u_{i} v_{i} z-\sum v_{i} z u_{i}=\sum\left[u_{i}, v_{i} z\right] \in[A, A]
$$


Hence we have $\operatorname{Ker}(s)=[A, A]$. Moreover,

$$
s^{2}(x)=s\left(\sum v_{i} x u_{i}\right)=s\left(\sum u_{i} v_{i} x\right)=s(x) .
$$

This means that $s$ is a projection. We have therefore a direct sum decomposition

$$
A=[A, A] \oplus s(A) .
$$

For such an $A$, we may substitute $s: A \rightarrow A$ for $\varepsilon: A \rightarrow A^{a}$, to the effect that traces and rank elements are elements of $A$ (In some important cases, $s(A)$ coincides with the center of $A$.).

4. Let $B$ be a ring and there be given a unitary homomorphism $\varphi: A \rightarrow B$. Assume that $B$, considered as a left $A$-module via $\varphi$, is finitely generated and projective. The right multiplication $b_{r}$ by $b \in B$ is an $A$-endomorphism of $B$. We call $\operatorname{Tr}\left(b_{r}\right)$ the trace of $b$ with respect to $A$, and denote it as $\operatorname{Tr}_{B / A}(b)$. $\operatorname{Tr}_{B / A}$ is a symmetric additive mapping $B \rightarrow A^{a}$. If $A$ is commutative and $\varphi(A)$ is in the center of $B$ (i.e. if $B$ is an algebra over $A$ ), $\operatorname{Tr}_{B / A}$ is an $A$-linear symmetric mapping $B \rightarrow A$.

1.2. Let $\varphi: A \rightarrow B$ be a unitary ring homomorphism. $\varphi$ induces a homomorphism $\varphi^{a}: A^{a} \rightarrow B^{a}$ such that $\varepsilon^{\prime} \circ \varphi:=\varphi^{a} \circ \varepsilon$, where $\varepsilon^{\prime}$ is the canonical epimorphism $B \rightarrow B^{a}$. If $P$ is a finitely generated projective left $A$-module, then $P^{\prime}=B \otimes{ }_{A} P$ is a finitely generated projective left $B$-module. The dual of $P^{\prime}$ is naturally identified with $\operatorname{Hom}_{A}(P, B)$. If $\theta\left(\sum \xi_{i} \otimes x_{i}\right)=1_{P}\left(x_{i} \in P, \xi_{i} \in P^{*}\right)$, then $\theta\left(\sum \varphi_{0}\right.$ $\left.\xi_{i} \otimes\left(1 \otimes x_{i}\right)\right)=1_{P^{\prime}}$. Now, let $f$ be an endomorphism of $P$. Then $1 \otimes f$ is an endomorphism of $P^{\prime}$, and we have immediately

$$
\operatorname{Tr}_{B}(1 \otimes f)=\varphi^{a} \operatorname{Tr}_{A}(f)
$$

In particular, we have

$$
r_{B}\left(B \otimes{ }_{A} P\right)=\varphi^{a} r_{A}(P) .
$$

Examples 5. Let $R$ be an integral domain, and $K$ its field of qutients. For a finitely generated projective $R$-module $P$, let $n$ be the dimension of the vector space $K \otimes P$ over $K$. Then we have $r_{R}(P)=n \cdot 1$.

6. Let $R$ be a commutative Noetherian ring, and $P$ a finitely generated projective $R$-module. Let $\mathrm{m}$ be a maximal ideal of $R, R_{\mathfrak{m}}$ the local ring at $\mathrm{m}$, and $\varphi_{\mathfrak{m}}$ the canonical homomorphism $R \rightarrow R_{\mathfrak{m}} . \quad P$ is said to be of rank $n$ if the free $R_{\mathfrak{m}}$-module $R_{\mathfrak{m}} \otimes P$ is of rank $n$ for every maximal ideal $m$. If this is the 
case, we have $\varphi_{\mathfrak{m}}\left(x_{R}(P)-n \cdot 1\right)=0$ for every $m$. It follows that $r_{R}(P)=n \cdot 1$.

We shall come back to the general case, and assume now that $B$ is finitely generated and projective as a left $A$-module. A finitely generated projective $B$-module $P$ is then a finitely generated projective $A$-module. Let $\theta \sum \lambda_{i} \otimes u_{i}=1_{B}$, $\left(u_{i} \in B, \lambda_{i} \in \operatorname{Hom}_{A}(B, A)\right)$. If $x_{j} \in P, \xi_{j} \in \operatorname{Hom}_{B}(P, B)$ are such that $\theta \sum \xi_{j} \otimes x_{j}$ $=1_{p}$, then $u_{i} x_{j} \in P$ and $\lambda_{i} \circ \xi_{j} \in \operatorname{Hom}_{A}(P, A)$ satisfy $\theta\left(\sum_{i, j} \lambda_{i} \circ \xi_{j} \otimes u_{i} x_{j}\right)=1_{p}$. We consider the following two particular cases:

i) Let $f \in \operatorname{Hom}_{B}(P, P)$. By definition, we have $\operatorname{Tr}_{B}(f)=\varepsilon^{\prime} \sum \xi_{j}\left(f\left(x_{j}\right)\right)$. If we regard $f \in \operatorname{Hom}_{A}(P, P)$, then, using the notation of Example 4, we have

$$
\begin{aligned}
\operatorname{Tr}_{A}(f)=\varepsilon \sum_{i, j} \lambda_{i} \xi_{j}\left(f\left(u_{i} x_{j}\right)\right) & =\varepsilon \sum_{i} \lambda_{i}\left(u_{i} \sum_{j} \xi_{j}\left(f\left(x_{j}\right)\right)\right) \\
& =\varepsilon \sum_{i} \lambda_{i}\left(u_{i} \operatorname{Tr}_{B}(f)\right)=\operatorname{Tr}_{B / A}\left(\operatorname{Tr}_{B}(f)\right) .
\end{aligned}
$$

This is the chain theorem:

$$
\operatorname{Tr}_{A}=\operatorname{Tr}_{B / A} \circ \operatorname{Tr}_{B}
$$

In particular, if $B$ is an algebra over a commutative ring $R$, and finitely generated and projective as an $R$-module, and if every element of $A^{a}$ has a representative in $R$, then we have

$$
\operatorname{Tr}_{R}(f)=r_{R}(B) \cdot \operatorname{Tr}_{B}(f),
$$

and in particular,

$$
r_{R}(P)=r_{R}(B) \cdot r_{B}(P) .
$$

ii) Let $A$ be a commutative ring, denoted by $R$ as above. Then the operation of $b \in B$ to $P$ is an endomorphism $\rho(b) \in \operatorname{Hom}_{R}(P, P)$, and we have

$$
\operatorname{Tr}_{R}(\mu(b))=\sum \lambda_{i} \xi_{j}\left(b u_{i} x_{j}\right)=\sum_{j} \lambda_{i}\left(b u_{i} \sum \xi_{j}\left(x_{j}\right)\right) .
$$

Using the natural homomorphism $\eta: B \otimes_{R} B^{\circ} \rightarrow \operatorname{Hom}_{R}(B, B)$ (where $B^{\circ}$ is an anti-isomorphic copy of $B$ corresponding to the right multiplications), this is described as

$$
\operatorname{Tr}_{R}(\rho(b))=\operatorname{Tr}_{R}\left(\eta\left(b \otimes r_{B}(P)^{\circ}\right)\right) .
$$

EXAMPLE 7. Let $B$ be the groupalgebra $R G$ of a finite group $G$ of order $g$ over a commutative ring $R$. We write the elements of $R G$ in the form $\sum_{\sigma \in G} a_{o} \sigma$, $\left(a_{\sigma} \in R\right)$. For a finitely generated $R$-projective $R G$-module $P$, we define the character $\chi_{P}$ by $\chi_{P}(\sigma)=\operatorname{Tr}_{R}(\sigma)$. Now assume further that $P$ is $R G$-projective, 
and let $r_{R G}(P)=\varepsilon\left(\sum s_{:} \tau^{-1}\right)$. Applying the above case ii), we have

$$
\chi_{P}(\sigma)=\operatorname{Tr}\left(\eta\left(\sigma \otimes\left(\sum s_{\tau} \tau^{-1}\right)^{0}\right)\right)=\sum_{\rho} s_{\mu}-t_{\sigma_{p}} .
$$

In particular, we have

$$
r_{R}(P)=\varkappa_{P}(1)=g s_{1}
$$

If $R$ is an integral domain with the quotient field $K$, and $n$ is the dimension of the vector space $K \otimes P$, then $r_{R}(P)=n \cdot 1$ (Example 5). Hence we have $g s_{1}=$ $n \cdot 1$. Let $K$ be of characteristic 0 , and let $g_{0}$ be the largest factor of $g$ which is a unit in $R$. Then $n$ must be divisible by $g / g_{0}$. For instance, if $K$ is an algebraic number field and $R$ its ring of integers, then $n$ is a multiple of $g$ (Nakayama [7]). In the local case that $R$ is the ring of $p$-adic integers, $p$ being a prime divisor of a rational prime $p, n$ is a multiple of the order of a Sylow $p$-subgroup of $G$.

If $g$ is a unit in $R$, then $R G$ is a separable algebra. A linear mapping $s$ : $A \rightarrow A$ of Example 3 is obtained in this case by

$$
s\left(\sum s_{\tau} \tau^{-1}\right)=\frac{1}{g} \sum_{\rho} \rho\left(\sum s_{\tau} \tau^{-1}\right) \rho^{-1}=\frac{1}{g} \sum_{\sigma}\left(\sum_{\rho} s_{\rho}-\sigma_{\rho}\right) \sigma^{-1} .
$$

( $s(R \hat{G})$ coincides with the center of $R G)$. Hence we have in the sense of Example 3

$$
r_{R \theta}(P)=\frac{1}{g} \sum_{\sigma} \chi_{P}(\sigma) \sigma^{-1}
$$

When the Krull-Schmidt theorem holds for projective $R G$-modules, then the above equality is nothing but the expression of central idempotents by means of characters (cf. Example 2). Obviously it is possible to derive properties of characters from properties of rank elements.

1.3. We can obviously speak of the traces and the rank elements for right modules.

Let $P$ be a finitely generated projective left $A$-module. By the duality, the bidual $P^{* *}$ is canonically identified with $P$. On the other hand, we have the anti-isomorphism $t: \operatorname{Hom}_{A}(P, P) \rightarrow \operatorname{Hom}_{A}\left(P^{*}, P^{*}\right)$ taking the transposed: $t_{f}(\xi)$ $(x)=\xi(f(x)) \quad\left(x \in P, \xi \in P^{*}\right)$. The diagram




is commutative. It follow's immediately

$$
\operatorname{Tr}(f)=\operatorname{Tr}\left({ }^{t} f\right) .
$$

In particular, $r(P)=r\left(P^{*}\right)$.

If $A$ has an anti-involution $a \rightarrow \bar{a}, P^{*}$ may be considered as a left $A$-module, by the new operation $a \hat{\xi}=\xi \bar{a}$. In this case, the identification of $P$ and $P^{* *}$ is realized by $x(\xi)=\overline{\xi(x)}$. Therefore we have

$$
\overline{\operatorname{Tr}(f)}=\operatorname{Tr}\left({ }^{t} f\right), \quad \overline{r(P)}=r\left(P^{*}\right)
$$

This applies, in particular, for the groupalgebra $A=R G$ of a finite group $G$ over a commutative ring $R$ and its canonical anti-involution $\bar{\sigma}=\sigma^{-1}(\sigma \in G)$.

\section{$\S 2$. Projective modules over an algebra over a complete local ring}

Let $\mathrm{D}$ be a complete local ring with maximal ideal $\mathrm{m}$, and $A$ an algebra over 0 finitely generated as an 0 -module. $A / \mathrm{m} A$ is a finite dimensional algebra over $\mathrm{o} / \mathrm{m}$. Let $N / \mathrm{m} A$ be the radical of $A / \mathrm{m} A$, and denote the natural epimorphism $A \rightarrow A / N$ by $a \rightarrow \bar{a}$. Since 0 is complete, every idempotent of $A / \mathrm{m} A$ can be lifted to $A$ Therefore, if $A e$ is an indecomposable left ideal of $A, e$ being an idempotent, then $A e / \mathrm{m} A e$ is also indecomposable and $A e / N e=A / N \bar{e}$ is irreducible. Further, for two primitive idempotents $e$ and $e^{\prime}, A e \cong A e^{\prime}$ if and only if $A e / \mathrm{m} A e$ $\cong A e^{\prime} / \mathrm{m} A e^{\prime}$, and the latter isomorphism holds if and only if $A / N \bar{e} \cong A / N \bar{e}^{\prime}$. (For these facts, see Curtis and Reiner [3].)

We have remarked in Example 2 that if $A e \cong A e^{\prime}$ then $\varepsilon(e)=\varepsilon\left(e^{\prime}\right)$. As a sort of converse to this, we shall prove

Lemma. Assume that $A / N$ is a direct sum of full matrix algebras over $\mathrm{o} / \mathrm{m}$ and that if the characteristic of $\mathrm{o} / \mathrm{m}$ is $p \neq 0$, then $A^{a}$ is $p$-torsionfree. If $e_{1}$, $\ldots, e_{r}$ are primitive idempotents of $A$ such that $A e_{1}, \ldots, A e_{r}$ are non-isomorphic to each other, then $\varepsilon\left(e_{1}\right), \ldots, \varepsilon\left(e_{r}\right)$ are $\mathrm{Z}$-linearly independent, $\mathrm{Z}$ being the ring of rational integers.

Proof. Suppose that there are non-zero integers $n_{1}, \ldots, n_{r}$ such that $\sum n_{i} \varepsilon\left(e_{\imath}\right)=0$. By assumption, when the characteristic of $\mathrm{o} / \mathrm{m}$ is $p \neq 0$, we may assume that one of $n_{i}$ 's, say $n_{1}$, is relatively prime to $p$. It follows that $\sum n_{i} \varepsilon$ $\left(\bar{e}_{i}\right)=0$, and we have $n_{1} \cdot \overline{1} \neq 0$. Let $A / N=\bar{A}_{1} \oplus \cdots \oplus \bar{A}_{s}$ be the decomposition of $A / N$ into simple components. Since $A / N \bar{e}_{i}, i=1, \ldots, r$, are non-isomorphic 
to each other, $\bar{e}_{i}$ 's are contained in different components, say $\bar{e}_{i} \in \bar{A}_{i}$. Since $(A / N)^{a}$ is the direct sum $\bar{A}_{1}^{a} \oplus \cdots \oplus \bar{A}_{s}^{a}$, and $n_{i} \varepsilon\left(\bar{e}_{i}\right) \in \bar{A}_{i}^{a}$, we must have $n_{i} \varepsilon\left(\bar{e}_{i}\right)=\theta$. In particular, $n_{1} \bar{e}_{1} \in\left[\bar{A}_{1}, \bar{A}\right]$. But this is impossible, since $\bar{A}_{1}$ is a full matrix algebra and $\bar{e}_{1}$ is a primitive idempotent.

THEOREM. Let $A$ be an algebra over a complete local ring 0 finitely generated as an $\mathrm{D}$-module, and if $\mathrm{o} / \mathrm{m}$ has the characteristic $p \neq 0$, we assume that $A^{a}$ is p-torsion free. Two finitely generated projective A-modules $P_{1}$ and $P_{2}$ are isomorphic if and only if $r_{A}\left(P_{1}\right)=r_{A}\left(P_{2}\right)$.

Proof. Let $L$ be an extension field of $\mathfrak{o} / \mathrm{m}$ of finite degree such that the semisimple residue class algebra of $A / \mathrm{m} A \otimes L$ is a direct sum of full matrix algebras over $L$. There exists a complete local ring $D^{\prime}$ such that

i) $\mathfrak{D}^{\prime}$ contains $\mathfrak{D}$ and is $\mathfrak{D}$-free of finite rank, and

ii) the maximal ideal of $\mathrm{o}^{\prime}$ is $\mathrm{mo}^{\prime}$ and $\mathrm{o}^{\prime} / \mathrm{mo}^{\prime} \cong L$ (cf. [1], [6], [8] etc.). Put $A^{\prime}=A \otimes_{D D^{\prime}}$. Then $A^{\prime} /\left[A^{\prime}, A^{\prime}\right] \cong A /[A, A] \otimes_{D D^{\prime}}$ and this is $p$-torsion free. Hence $A^{\prime}$ satisfies the assumptions on $A$ in the above Lemma. By the KrullSchmidt theorem, $P_{1} \cong P_{2}$ if and only if $P_{1} \otimes \mathcal{D}^{\prime} \cong P_{2} \otimes \mathcal{D}^{\prime}$, while $r_{A}\left(P_{1}\right)=r_{A}\left(P_{2}\right)$ if and only if $r_{A^{\prime}}\left(P_{1} \otimes \mathcal{O}^{\prime}\right)=r_{A^{\prime}}\left(P_{2} \otimes \mathcal{O}^{\prime}\right)$. We may therefore assume that $A$ itself already satisfies the assumptions of Lemma. Now, a projective module is isomorphic to a direct sum of indecomposable left direct summands of $A$. Let $A e_{1}, \ldots, A e_{s}$ be a complete set of representatives of the isomorphism classes of indecomposable left direct summands of $A$, and let $m_{i}, n_{i}$ be the multiplicity of $A e_{i}$ in $P_{1}, P_{2}$, respectively, $i=1, \ldots, s$. By Example 2, we have

$$
r_{A}\left(P_{1}\right)=\sum m_{i} \varepsilon\left(e_{i}\right), \quad r_{A}\left(P_{2}\right)=\sum n_{i} \varepsilon\left(e_{i}\right) .
$$

If $r_{A}\left(P_{1}\right)=r_{A}\left(P_{2}\right)$, we have $\sum\left(m_{i}-n_{i}\right) \varepsilon\left(e_{i}\right)=0$. Applying the above Lemma, we have $m_{i}=n_{i}, i=1, \ldots, s$. This means $P_{1} \cong P_{2}$. The converse is trivial.

Corollary. Let o be a complete local domain of characteristic 0 with quotient field $K$, and let $A$ be an algebra over o finitely generated as an $\mathrm{D}$-module. Assume $A^{a}$ is D-torsionfree. Then two finitely generated projective $A$-modules $P_{1}$ and $P_{2}$ are isomorphic if and only if $K \otimes P_{1}$ and $K \otimes P_{2}$ are isomorphic.

Proof. Let $\varphi$ be the canonical injection $\mathfrak{D} \rightarrow K$. The assumption that $A^{a}$ is o-torsionfree means that $\varphi^{a}: A^{a} \rightarrow(K \otimes A)^{a}$ is a monomorphism. Now, if $K \otimes P_{1}$




that $r_{A}\left(P_{1}\right)=r_{A}\left(P_{2}\right)$. Since our $A^{a}$ is $\mathbf{Z}$-torsionfree, we may apply the above Theorem, and conclude that $P_{1}$ and $P_{2}$ are isomorphic. The converse is clear.

Remark. In the above, we only used the $p$-torsionfreeness and $p$-torsionfreeness of the group generated by all $\varepsilon(e)$, ( $e$ idempotents), not of the whole group $A^{a}$. For instance, let $\mathfrak{D}$ be the ring of 2 -adic integers, and $A$ the usual quaternion algebra over $\mathfrak{b}$, with the basis $1, i, j, k$. Then $[A, A]=2(\mathfrak{o} i+\mathfrak{o} j+\mathrm{o} k)$, so that $A^{a}$ has non-trivial 2-torsion. But the only idempotent in $A$ is 1 , and $\varepsilon(1)$ is $D$-free. Hence we may apply the above arguments to this case (This case is rather trivial as every finitely generated projective $A$-module is free.).

Example 8. Let $A$ be the groupalgebra of a finite group $G$ over a complete local domain 0 of characteristic $0 . A^{a}$, being 0 -isomorphic to the center of $A$, is D-torsionfree. Therefore Theorem and its Corollary can be applied to this case. The latter is nothing but the characteristic zero case of Swan's local theorem [9].

\section{REFERENCES}

[1] M. Auslander and O. Goldman, The Brauer group of a commutative ring, Trans. Amer. Math. Soc., 97 (1960), 367-409.

[2] H. Bass, Projective modules over algebras, Ann. of Math., 73 (1961), 532-542.

[3] C. Curtis and I. Reiner, Representation theory of finite groups and associative algebras, Interscience, 1962.

[4] I. Giorgiutti, Modules projectifs sur les algèbres de groupes finis, C. R. Paris, 250 (1960), 1419-1420.

[5] A. Hattori, Integral representations and projective modules, Proceedings of the Symposium on Group Theory, Tokyo, (1961), 95-120 (in Japanese).

[6] A. Hattori, Semisimple algebras over a commutative ring, J. Math. Soc. Japan, 15 (1963), 404-419.

[7] T. Nakayama, On modules of trivial cohomology over a finite group II, Nagoya Math. J., 12 (1957), 171-176.

[8] J.-P. Serre, Corps locaux, Hermann, 1962.

[9] R. Swan, Induced representations and projective modules, Ann. of Math., 71 (1960), $552-578$.

Tokyo University of Education 